\title{
Numerical optimisation of the charge/discharge characteristics of wayside energy storage systems by the embedded simulation technique using the railway power network simulator RTSS
}

\author{
K. Shiokawa \& R. Takagi \\ Department of Electrical Engineering, \\ Kogakuin University, Japan
}

\begin{abstract}
Railways have been believed to be the best mode of transport in terms of energy consumption. However, because other modes of transport are rapidly catching up and improving energy efficiency, the railway industry is now keen to reduce its energy consumption. Recent years have seen the active development of high performance secondary batteries for pure and hybrid electric vehicles. It is believed that these batteries can be applied to electric railways to reduce energy consumption. The ESSs (Energy Storage Systems), in which batteries are used as storage media, can be installed somewhere in the power supply network for railways to charge electric energy when there is excessive regenerated power in the network. These ESSs can also be used to assist the supply substations when there is a high power demand. ESSs can be installed either as stationary wayside installations or as added equipment on-board the running trains. In this paper, the wayside installation type will be discussed.

In Japan, several ESS installations have already been commissioned and are in use within the working railway power networks. However, the "properness" of their charge/discharge characteristics has not been actively evaluated. It is believed that small changes in the voltage-current characteristics of an ESS may have a significant impact on the actual effect of the introduction of the ESS itself.

The effects of the introduction of ESSs can be evaluated using the multi-train power network simulation program. Once the characteristics of the ESSs are
\end{abstract}


given, the evaluation can be made using the simulation program. Therefore, by embedding the simulation program in the numerical optimisation process, the optimal ESS characteristics can be obtained.

In this paper, the authors' efforts to use this embedded simulation technique to optimise the charge/discharge characteristics of the ESS are described in detail.

Keywords: optimisation, DC power feeding networks for electric railways, energy storage systems, multi-train power feeding network simulator, electric railways.

\section{Introduction}

Thanks to the recent developments of high performance secondary battery technologies for pure electric vehicles (EVs) or hybrid electric vehicles (HEVs), it is now expected that the energy storage systems (ESSs) will contribute to the reduction in energy consumption of electric railways, mainly their DC variants.

By introducing one or more ESSs somewhere in the power feeding network of an electric railway, its energy consumption can be reduced in the following way:-

(1) By charging energy when there is excessive regenerative power in the feeding network; and

(2) By discharging energy when there is heavy load in the feeding network.

Method (1) intends to assure that there is always enough line receptivity for any regenerating train so its regenerative brake performance is always fully utilised. Method (2) makes use of the energy acquired by method (1), reduces feeding losses and suppresses high peak currents at the feeding substations.

However, since the energy and power capacity of an ESS is generally limited, there is a strong need for improved charge/discharge control to obtain maximum effects by the introduction of smallest possible ESSs.

In this paper, the authors describe the results of the preliminary study on the optimisation of parameters for the charge/discharge control of the ESSs by means of the embedded simulation using the detailed multi-train power network simulator "RTSS" which incorporates the ESS simulation model.

\section{Voltage-based charge/discharge control of ESSs}

An ESS for the DC electric railways consists of a power electronic converter and the energy storage devices (ESDs) in which the electric energy is stored. Despite the recent developments, ESDs are generally expensive and therefore the capacity of the ESS to be introduced into the railway power network must be minimised. For this purpose, the optimisation of the charge/discharge control characteristics is important.

There are various charge/discharge control schemes that can be used to control the wayside-installed ESSs. In this paper, the authors assume the charge/discharge control based on the line voltage at the point where the ESS is 
connected to the railway power feeding network. This section is dedicated to the detailed description of this control scheme.

\subsection{Current-voltage (I-V) characteristics}

In the voltage-based charge/discharge control of an ESS, its charge/discharge current is determined by the line voltage at the connecting point of the ESS. The $\mathrm{I}-\mathrm{V}$ characteristics assumed in this paper are shown in Fig. 1. Under this scheme, the ESS acts as follows:

(1) If the voltage of the connecting point is below $\mathrm{V}_{c}$, the ESS discharges power so the voltage drop is reduced. If the voltage is below $V_{b}\left(<V_{c}\right)$, then the discharge current is set to the maximum value of $I_{d m a x}$. Above $V_{b}$, the discharge current decreases as the voltage increases.

(2) If the voltage of the connecting point is above $V_{d}$, the ESS charges power so the line becomes receptive for the regenerating trains. If the voltage is above $V_{e}\left(>V_{d}\right)$, then the discharge current is set to the maximum value of $I_{c m a x}$ (i.e. the charge current is set to $-I_{c \max }$ ). Below $V_{e}$, the charge current decreases as the voltage decreases.

(3) If the voltage of the connecting point is between the above two regions, the ESS enters the floating mode, in which the ESS either charges or discharges power to return to the pre-defined state of charge (SOC). The discharge current during this floating mode, $\mathrm{I}_{\mathrm{f}}$, is determined by the current $\mathrm{SOC}$ value of the system.

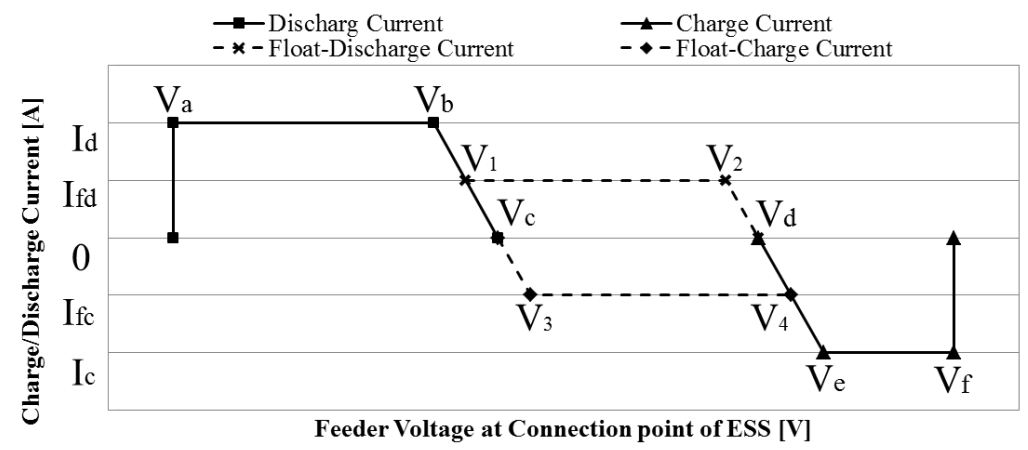

Figure 1: Current-voltage characteristic of ESSs.

\subsection{Current-SOC characteristics}

Using the characteristics defined in Section 2.1, it may happen that the ESS exceeds the safe operating range by either charging or discharging too much energy. In order to prevent this, the current-SOC characteristics as shown in Fig. 2 give the parameters $I_{d \max }, I_{c \max }$ and $I_{f}$ according to the current SOC of the ESS. 


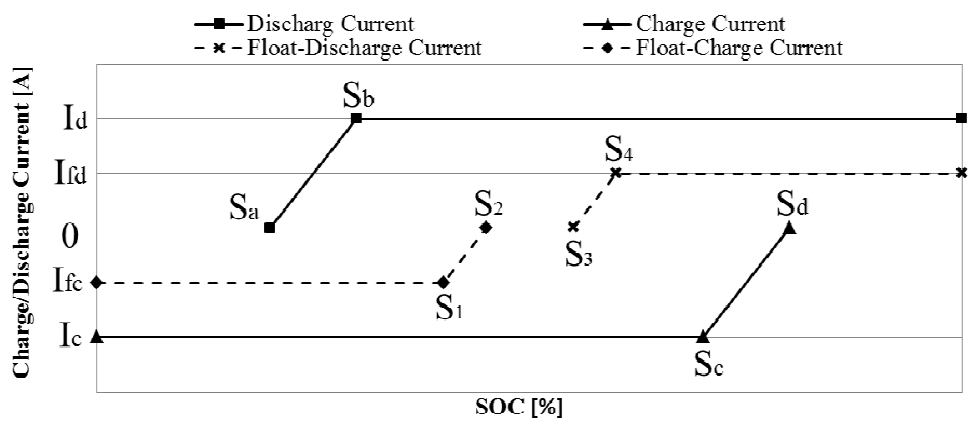

Figure 2: $\quad$ Current-SOC characteristic of ESSs.

\subsection{Parameters to be optimised}

In Figs. 1 and 2, there are a number of parameters that determine the performance of the system. In the following, the set of parameters listed hereunder is to be optimised:-

- $\mathrm{V}_{\mathrm{b}}, \mathrm{V}_{\mathrm{c}}, \mathrm{V}_{\mathrm{d}}$ and $\mathrm{V}_{\mathrm{e}}$ in Fig. 1,

- $\mathrm{S}_{1}, \mathrm{~S}_{2}, \mathrm{~S}_{3}$ and $\mathrm{S}_{4}$ in Fig. 2, and

- $\mathrm{I}_{\mathrm{dmax}}, \mathrm{I}_{\mathrm{cmax}}$ and $\mathrm{I}_{\mathrm{f}}$ in both figures.

\section{The optimisation technique}

\subsection{Embedded simulation}

Optimisation requires iteration of the following tasks: (1) designing of the system parameters, (2) simulation, and (3) evaluation. If one set of the tasks (1) through (3) is done, a slightly different design is given, and the same process is repeated until the best design is found.

Generally, in steps (2) and (3) above, the simplified simulation model is used to shorten the calculation time required for the entire optimisation process. However, the authors' research group has developed and maintained the detailed multi-train power network simulation program. Except for the concern about calculation times, it can be generally expected that embedding the detailed model in the optimisation process yields better results.

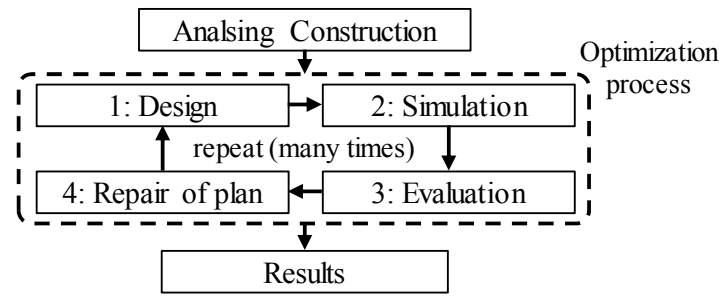

Figure 3: The flowchart of optimisation processes. 


\subsection{Multi-train railway power network simulator "RTSS"}

"RTSS" is a multi-train simulator of the power feeding network for the DC electric railway [1]. First developed in 1990, it has been maintained and used extensively within the authors' research group. This program incorporates the detailed simulation model, including the voltage-dependent train performance model. Inclusion of the voltage-dependency of train performance, however, would generally mean that the start-to-stop times of trains may fluctuate when other parameters such as substation output voltages change, resulting in unreliable evaluation of energy consumption. RTSS resolves this issue by implementing a small subprogram that auto-calculates the time at which any train in the simulation ceases acceleration, enabling the simulation with precise start-to-stop time of any inter-station run in the simulation. This program has actually been used for evaluation of a number of railway projects around Japan.

In the numerical optimisation presented in this paper, RTSS is used to calculate the evaluation function.

\subsection{The details of the evaluation function}

The fitness of the set of parameters to be optimised, shown in Section 2.3, is to be determined by the output yielded by the RTSS simulation. Out of the various evaluation results RTSS outputs, the most important is the substation total input energy, which is the aggregated energy that flows through all feeding substations from the utility power network to the railway feeding network in the system to be simulated; this shows the energy consumption of the railway itself, and is therefore used as the basis of the evaluation function used in this paper.

The substation total input energy may include the energy that comes from, or goes into, the ESSs in the system. Because RTSS assumes that the train timetables be cyclic, the trajectory of the SOC of any ESS in the system to be simulated is generally expected to be cyclic after a long time. However, it may happen that the SOC of the ESS at the start and end of the simulation may differ; therefore, this is taken into consideration as penalty in the evaluation function.

The evaluation function used in the optimisation is as follows:-

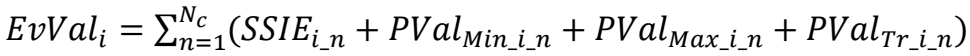

$$
\begin{aligned}
& \text { PVal }_{\text {Min_i_n }}=C_{1}\left(\operatorname{RefSOC} C_{\text {Min }}-\text { MinSOC }_{i_{-} n}\right) \\
& \text { PVal }_{\text {Max_inn }}=C_{1}\left(\text { MaxSOC }_{i_{-} n}-\operatorname{RefSOC} C_{\text {Max }}\right) \\
& P V a l_{T r_{-} i_{-} n}=C_{2}\left(\mid \operatorname{IntSOC}_{i_{-} n}-\text { FinSOC }_{i_{-} n} \mid-\operatorname{RefSOC}_{\operatorname{Tr}}\right) \\
& C_{1}=\log _{10} S S I E_{0} \\
& C_{2}=1+\log _{10} S S I E_{0} \\
& \text { FinVal }=\frac{2\left(E v V a l_{\text {max }}-E v V a l_{\text {min }}\right)}{E v V a l_{\max }+E v V a l_{\text {min }}} \\
& i \text { : Index of solutions } \\
& n \text { : Index of train opelation case } \\
& \text { EvVal: Evaluation value }
\end{aligned}
$$


SSIE : Total substation input energy

PVal: Penalty value

RefSOC : Reference SOC value (upper or lower limit)

MinSOC $_{i}$ : Minimum SOC value

$\operatorname{MaxSOC}_{i}$ : Maximum SOC value

$\operatorname{IntSOC}_{i}$ : Initial SOC value

FinSOC $_{i}$ : Final SOC value

FinVal : Criteria for the termination of optimisation

$C_{1}, C_{2}$ : Cost value

\subsection{Optimisation algorithm}

The multi-dimensional downhill simplex method (also called the Nelder-Mead method or the amoeba method) was used in this paper [2]. This algorithm utilises a polyhedron (simplex) with the $n+1$ vertices in $n$-th dimensional space, when $n$ variables are in the parameter set to be optimised. The position of any one vertex of the simplex corresponds to a set of values for the parameter set, and therefore a simplex as a whole represents a set of candidates of the optimal solution. During the optimisation process, the shape of the simplex is transformed according to the evaluation of the vertices until all the vertices are brought very close to the vertex with the best evaluation. Fig. 4 shows the flowchart of the downhill simplex method.

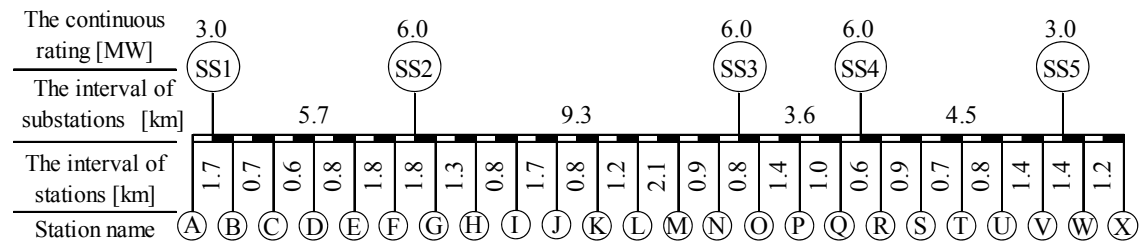

Figure 4: Substation and station displacements of the railway line for the case-study.

It is important to note that the simplicity of this optimisation method makes it easy to embed any detailed simulation tool in the process; another example of using the same algorithm is presented by Takagi et al. [2].

\section{Assumptions for the optimisation}

A set of data used in the case study optimisation by means of the proposed technique is generated based on anactual railway in Japan, the details of which is shown hereunder. 


\subsection{Details of the railway line}

The model railway line for the case-study is a metro-type commuter railway with 24 stations and the track length of 26.5 kilometres. All trains call at all stations en-route. There are 5 feeding substations, with the capacity of 3.0 MW (two substations located towards both ends of the line) and 6.0 MW (three substations located between two other substations). The gradient profile and other details of the line are shown below:-

Table 1: $\quad$ Other details of the railway line for the case-study.

\begin{tabular}{|c|c|c|c|}
\hline \multirow{7}{*}{ Route } & \multicolumn{2}{|c|}{ Number of stations } & 24 \\
\hline & \multicolumn{2}{|c|}{ Length of route $[\mathrm{km}]$} & 26.5 \\
\hline & \multicolumn{2}{|c|}{ Top speed of train $[\mathrm{km} / \mathrm{h}]$} & 85.0 \\
\hline & \multicolumn{2}{|c|}{ Feeder resistance $[\Omega / \mathrm{km}]$} & 0.03 \\
\hline & \multicolumn{2}{|c|}{ Type of electrified } & $\begin{array}{c}\text { DC1.5kV, } \\
\text { Double-tracks }\end{array}$ \\
\hline & \multirow{2}{*}{$\begin{array}{l}\text { Train per-hour } \\
\text { frequency }\end{array}$} & Off-peak [trains/h] & 10 \\
\hline & & Rush time [trains/h] & 5 \\
\hline \multirow{5}{*}{ Substation } & \multicolumn{2}{|c|}{ Number of substations } & 5 \\
\hline & \multicolumn{2}{|c|}{ Continuous rating [MW] } & $\begin{array}{l}3.0 \text { (route end), } \\
6.0 \text { (middle) }\end{array}$ \\
\hline & \multicolumn{2}{|c|}{ Type of substation } & Diode rectified \\
\hline & \multicolumn{2}{|c|}{ Voltage regulation $[\%]$} & 6.0 \\
\hline & \multicolumn{2}{|c|}{ Output voltage at non load [V] } & 1590.0 \\
\hline \multirow{4}{*}{ ESS } & \multicolumn{2}{|c|}{ Number of ESS to be installed in the line } & 1 \\
\hline & \multicolumn{2}{|c|}{ Position of the ESS $[\mathrm{km}]$} & $\begin{array}{c}11.8 \text { (between } \mathrm{J} \\
\text { and } \mathrm{K} \text { ) }\end{array}$ \\
\hline & \multicolumn{2}{|c|}{ Continuous rating [MW] } & 1.0 \\
\hline & \multicolumn{2}{|c|}{ Energy capacity [kWh] } & 200.0 \\
\hline \multirow{4}{*}{ Train } & \multirow{2}{*}{ Maximum power } & Powering[MW] & 2.9 \\
\hline & & Regenerating[MW] & 3.8 \\
\hline & \multirow{2}{*}{ Congestion } & Off-peak [\%] & 100.0 \\
\hline & & Rush time [\%] & 200.0 \\
\hline
\end{tabular}

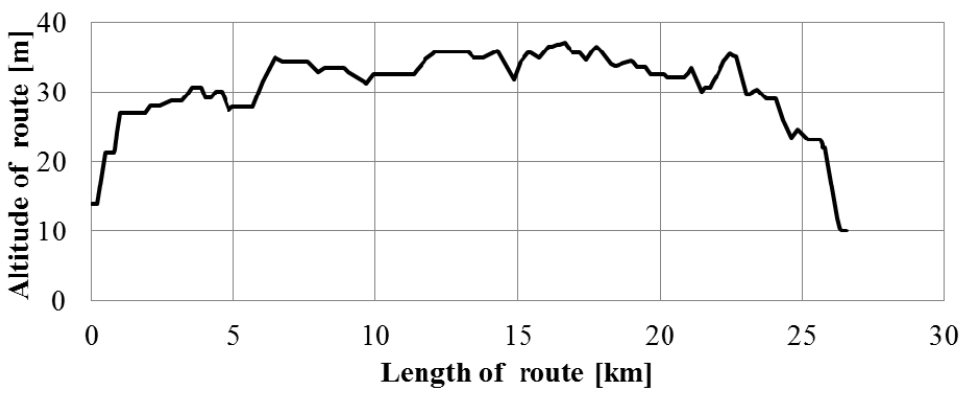

Figure 5: The gradient profile of the railway line for the case-study. 


\subsection{Details of other parameters used in the optimisation}

In this case-study, the set of parameters shown in Section 2.3 is to be optimised. In addition, the penalty value is added to the evaluation value when the trajectory of SOC of any ESS exceeds the range between RefSOC $\mathrm{Min}_{\text {and }}$ RefSOC $\mathrm{Cax}_{\mathrm{Max}}$ as shown in Table 2. RefSOC $\mathrm{Tr}_{\mathrm{r}}$ in the third row of Table 2 is to be used for calculation of Equation (4) in Section 3.3. Optimisation algorithm will terminate when FinVal of Equation (7) in Section 3.3 becomes lower than the "condition of optimisation termination" value shown in the last row of Table 2.

Table 2: $\quad$ Restrictions of optimisation.

\begin{tabular}{|r|l|}
\hline Upper limit of SOC $\left(\operatorname{RefSOC} C_{\operatorname{Max}}\right)[\%]$ & 25.0 \\
\hline Lower limit of SOC $\left(\operatorname{RefSOC} C_{\operatorname{Min}}\right)[\%]$ & 55.0 \\
\hline Deviation band of SOC $\left(\operatorname{RefSOC} C_{T r}\right)[\%]$ & 1.0 \\
\hline Condition of optimisation termination & $1.0 \mathrm{E}-6$ \\
\hline
\end{tabular}

\section{Results and discussions}

\subsection{The results}

The optimisation result was obtained after 4483 iterations, and it took 74 hours 43 minutes to complete the whole process. Tables 3 and 4 show the performance of power feeding network obtained by optimisation assuming off-peak and peak hour conditions, respectively.

Table 3: Comparison of the evaluations before and after the introduction of the ESS (off-peak-hour condition).

\begin{tabular}{|c|c|c|c|}
\hline Evaluated item & w/o ESS & w/ ESS & Diff. \\
\hline Regenerated energy $[\mathrm{kWh} / \mathrm{h}]$ & 1722.7 & 1819.5 & 96.8 \\
\hline Train consumed energy $[\mathrm{kWh} / \mathrm{h}]$ & 4483.7 & 4384.2 & 99.4 \\
\hline Feeder loss [kWh/h] & 262.9 & 248.8 & 14.1 \\
\hline Substation total input energy $[\mathrm{kWh} / \mathrm{h}]$ & 4746.6 & 4633.1 & 113.5 \\
\hline Regeneration rate $[\%]$ & 36.1 & 38.1 & 2.1 \\
\hline Bottom voltage of accelerating trains $[\mathrm{V}]$ & 1280.0 & 1324.2 & 44.2 \\
\hline
\end{tabular}

Table 4: Comparison of the evaluations before and after the introduction of the ESS (peak-hour condition).

\begin{tabular}{|c|c|c|c|}
\hline Evaluated item & w/o ESS & w/ ESS & Diff. \\
\hline Regenerated energy $[\mathrm{kWh} / \mathrm{h}]$ & 4320.7 & 4347.7 & 27.0 \\
\hline Train consumed energy $[\mathrm{kWh} / \mathrm{h}]$ & 7940.6 & 7904.2 & 36.4 \\
\hline Feeder loss [kWh/h] & 568.1 & 545.4 & 22.6 \\
\hline Substation total input energy $[\mathrm{kWh} / \mathrm{h}]$ & 8508.6 & 8449.7 & 58.9 \\
\hline Regeneration rate $[\%]$ & 45.9 & 46.3 & 0.3 \\
\hline Bottom voltage of accelerating trains $[\mathrm{V}]$ & 1288.6 & 1289.9 & 1.3 \\
\hline
\end{tabular}


Figs. 6 and 7 show the charge/discharge characteristics of the ESS obtained by optimisation.

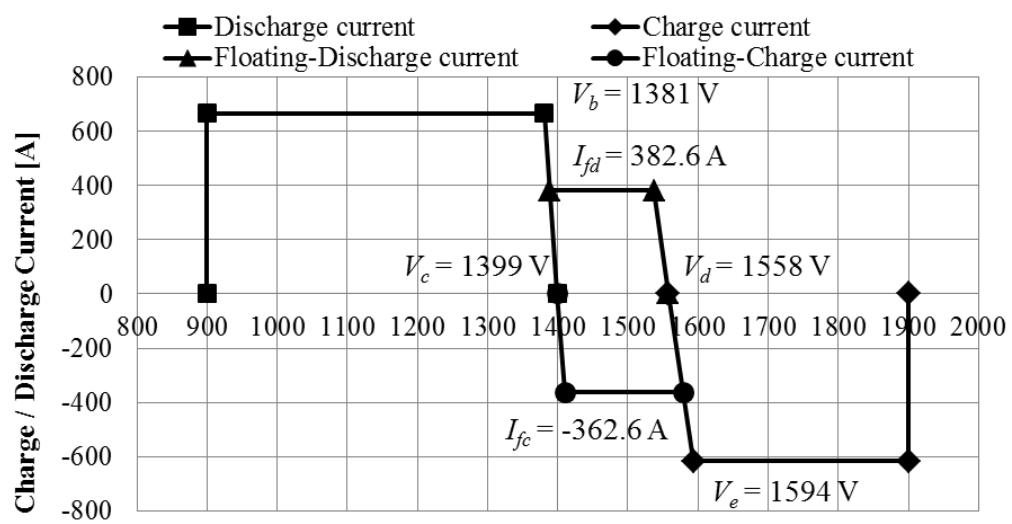

Voltage of the connecting point $[\mathrm{V}]$

Figure 6: The optimised current-voltage (I-V) characteristics of the ESS.

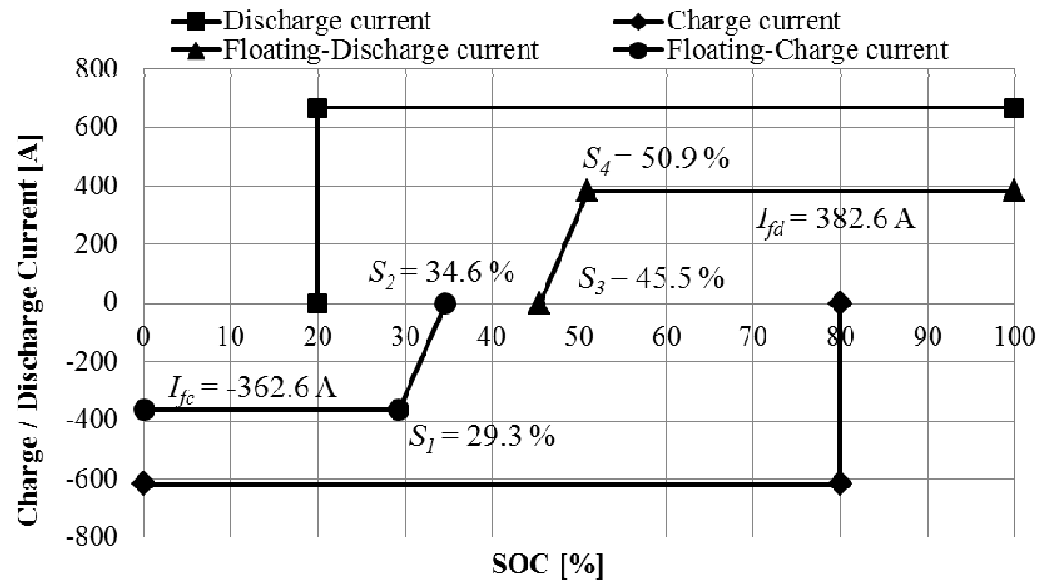

Figure 7: The optimised current-SOC characteristics of the ESS.

These results satisfy restrictions of optimisation as described in section 4.2.

\subsection{Discussions}

Under the off-peak-hour assumptions, the substation total input energy is reduced by $113.5 \mathrm{kWh} / \mathrm{h}$ by the introduction of the optimised ESS. This is mainly because ESS received the regenerating energy from the regenerative braking trains; it can be seen in Table 3 that, after the introduction of the ESS, the regenerated energy increases and the feeder loss decreases. Also, the SOC trajectory shown in Table 5 is very close to cyclic, minimising the penalty in the 
optimisation function. This result is what can be expected from the assumptions given in Sections 3.3 and 4.2 .

Table 5 shows the SOC trajectory of 2 train operating cases.

Table 5: $\quad$ SOC trajectory of the ESS.

\begin{tabular}{|c|c|c|c|c|c|}
\hline $\begin{array}{c}\text { Assumed time of } \\
\text { day }\end{array}$ & Unit & $\begin{array}{c}\text { Initial } \\
\text { SOC }\end{array}$ & $\begin{array}{c}\text { Maximum } \\
\text { SOC }\end{array}$ & $\begin{array}{c}\text { Minimum } \\
\text { SOC }\end{array}$ & $\begin{array}{c}\text { Final } \\
\text { SOC }\end{array}$ \\
\hline off-peak-hour & $\%$ & 52.0 & 47.8 & 53.0 & 52.1 \\
\hline peak-hour & $\%$ & 46.3 & 45.9 & 47.9 & 46.4 \\
\hline
\end{tabular}

Under the peak-hour assumptions, the improvement of the substation total input energy by the introduction of the ESS is $58.9 \mathrm{kWh} / \mathrm{h}$. This result can be explained as follows:-

(1) Little difference is observed for the regenerated energy evaluations, because the line receptivity is high even without the ESS under the peakhour condition.

(2) Feeder loss and the energy consumed by trains are reduced by the introduction of the ESS because it contributed to the prevention of the drop of line voltage.

\section{Conclusion}

The authors have proposed the numerical optimisation of the charge/discharge characteristics of the wayside ESS by the embedded simulation technique using the detailed simulation program "RTSS". Since RTSS is the detailed simulation program with proven track record, the results yielded by this technique is expected to be more reliable than the optimisation results using the simplified simulation models.

It must be pointed out, however, that the multi-dimensional downhill simplex method is not expected to reach the global optimum efficiently, and frequently the algorithm stops when it reaches a local minimum. Application of other algorithms, such as a GA (Generic Algorithm), must be considered in order to reach global optimum more reliably.

\section{References}

[1] R. Takagi: "The Development of the Integrated Intelligent System of Railway Power Feeding and Train Dispatching Subsystems for DC Electric Railways" (in Japanese), The University of Tokyo (PhD Thesis), 1995.

[2] R. Takagi, C. J. Goodman, C. Roberts: "Optimization of train departure times at an interchange considering passenger flows", Proc. IMechE Vol. 220 Part F, 2006.

[3] Press, W. H., et al.: "Numerical Recipes in C", Cambridge University Press, 1992.

[4] R. Takagi, T. Amano: "Evaluating On-board Energy Storage System Using Multi-train Simulator RTSS", The $4^{\text {th }}$ International Conference on Railway Traction Systems (RTS 2010), 2010. 